This item was submitted to Loughborough's Research Repository by the author.

Items in Figshare are protected by copyright, with all rights reserved, unless otherwise indicated.

\title{
Geometric elements for tolerance definition in feature-based product models
}

PLEASE CITE THE PUBLISHED VERSION

PUBLISHER

(c) Taylor and Francis

VERSION

AM (Accepted Manuscript)

LICENCE

CC BY-NC-ND 4.0

REPOSITORY RECORD

Gao, James, Keith Case, and N. Gindy. 2019. "Geometric Elements for Tolerance Definition in Feature-based Product Models”. figshare. https://hdl.handle.net/2134/13595. 
This item was submitted to Loughborough's Institutional Repository (https://dspace.lboro.ac.uk/) by the author and is made available under the following Creative Commons Licence conditions.

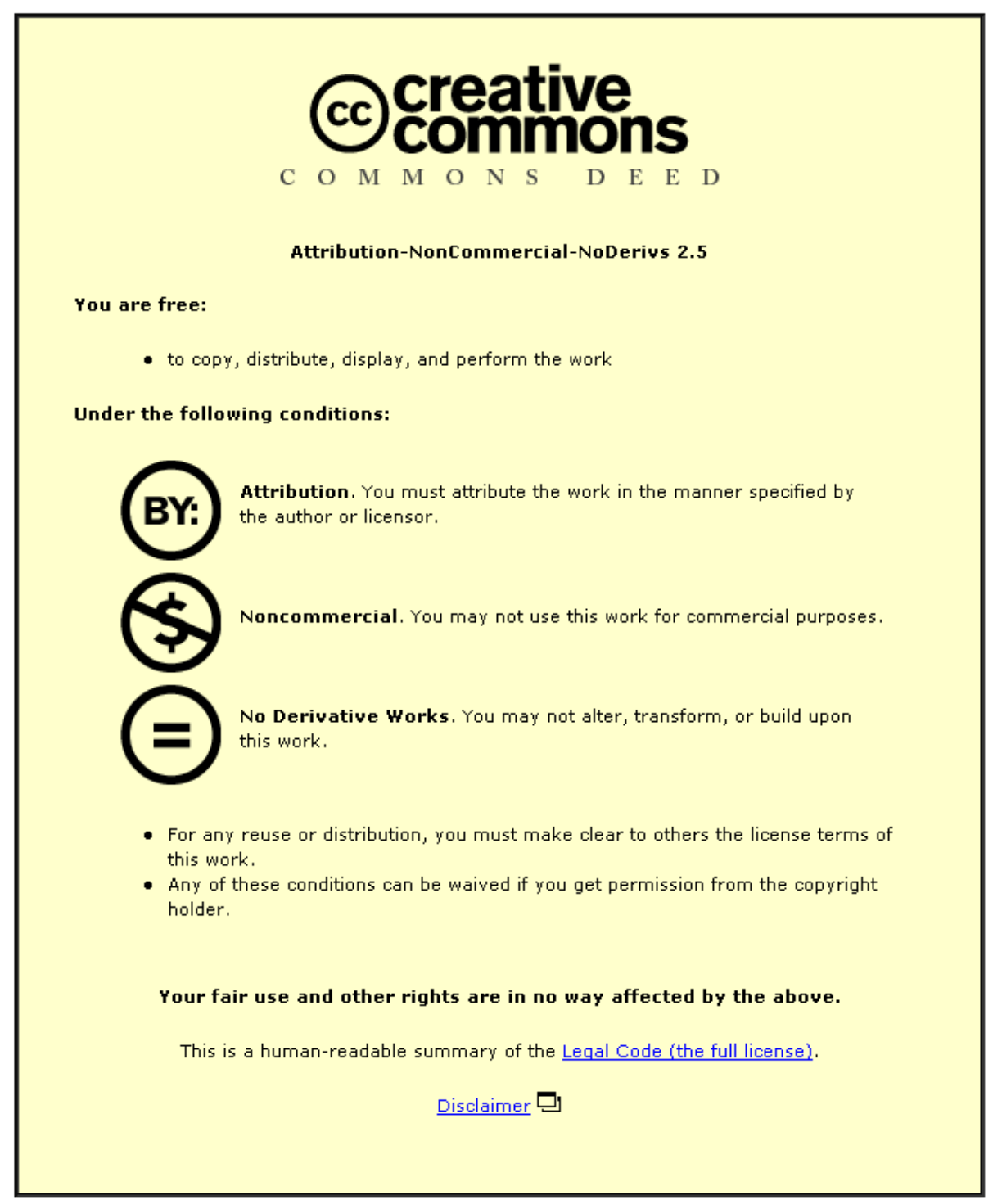

For the full text of this licence, please go to: http://creativecommons.org/licenses/by-nc-nd/2.5/ 
Gao, J., Case, K. and Gindy, N. (1994).

Geometric elements for tolerance definition in feature-based product models.

In K. Case, \& S. T. Newman (Eds.), 'Advances in Manufacturing Technology VIII', the Proceedings of the Tenth National Conference on Manufacturing Research,

NCMR 1994 (pp. 264-268). Loughborough University of Technology,

Loughborough, UK: London, UK, 5-7 September 1994: Taylor \& Francis.

\title{
GEOMETRIC ELEMENTS FOR TOLERANCE DEFINITION IN FEATURE-BASED PRODUCT MODELS
}

\section{Dr James Gao†, Dr Keith Case* and Professor Nabil Gindy**}

\author{
†School of Industry and Manufacturing Science, Cranfield University, Cranfield \\ Bedford, MK43 OAL \\ * Department of Manufacturing Engineering, Loughborough University of Technology \\ Loughborough, Leicestershire, LE11 3TU \\ **Department of Manufacturing Engineering and Operations Management, University of \\ Nottingham, Nottingham, NG72RD
}

\begin{abstract}
Product modelling is an essential part of all computerised design and manufacturing activities. A precise mathematical model of the geometry of products is important, but must be supplemented with technological information such as the material, mechanical properties, functional specifications and tolerances. Modern CAD systems can model and manipulate components with complex geometry. However, technological information is represented as text symbols on the computer screen or drawing, and subsequent application programs are frequently unable to use this information effectively. This paper discusses this problem, and establishes the geometric elements required for the representation or dimensions and tolerances in a feature-based product modelling environment.
\end{abstract}

\section{Introduction}

Engineering drawings are the traditional way to represent product information including the nominal geometry, dimensions and tolerances and other technological specifications, such as the mechanical properties of materials and method or heat treatment, initial status of stock (e.g. cast, forged or pre-cut), functional .and structural requirements. Drawings are produced by the design team and passed to the production engineers who interpret the information to find appropriate manufacturing processes. The tasks involved in such a traditional design and manufacturing procedure are increasingly being replaced by computerised systems (CAD/CAM).

The modelling and manipulation of the nominal geometry of components by computers is a successful and mature technology. However, the technological information, which is provided as annotation of the geometry of components in traditional drawings, has not been successfully represented together with the geometry of components in a single integrated form. In fact, the technological information is represented as text symbols on the computer screen or on the drawing output from the computer. Subsequent application programs are unable to use this information effectively (Juster, 1992). This problem, especially the representation of tolerances in the geometric model, becomes increasingly important as the product data model is recognised as playing a vital role in a fully integrated and concurrent computer aided engineering context. 
There are basically three types of 3 dimensional geometric models of components, i.e. wireframe, surface and solid models. Solid modelling is said to be the most "complete" representation of component geometry, in the sense that all required geometric information can be obtained from the model either directly or by simple calculation. There are two types or solid modelling, i.e. Constructive Solid Geometry (CSG) or set-theoretic and Boundary Representation (Brep) (Mantyla, 1988). A Brep model is based on a face-edge-vertex adjacency graph which contains more detailed and more explicit information than any other geometric representation. Even so, a Brep model is still not suitable for representing tolerances and other important technological information. This problem is discussed in the following sections.

\section{Geometric Elements Required for Tolerance Definition}

\section{Dimensions and tolerances}

There are three types of dimensions, i.e. functional, non-functional and auxiliary. Functional dimensions are essential to the function of an object and are potential candidates for tolerances. Non-functional and auxiliary dimensions would not normally be toleranced, as they are not essential to the function of the object. Tolerances which are applied to dimensions are called dimensional tolerances, and may be linear or angular.

Geometrical tolerances arc used to control the variation in the form of features. A geometrical tolerance defines the size and shape of a tolerance zone within which the feature is to lie. There are four types of geometrical tolerances, i.e. tolerances of form (straightness, flatness, roundness, cylindricity, profile of a line, profile of a surface), tolerances or attitude (parallelism, squareness, angularity), tolerances of location (position, concentricity, symmetry) and composite tolerance run-out).

\section{Features for tolerancing}

The basic geometric elements required for tolerance definition are features. Examples of features are surfaces (planar, cylindrical, spherical, conic and free form surfaces), lines (linear and curved) and points. These features are also known as single features as they are the most elementary building blocks or components. Note that surface features may be median planes of other features, line features may be axes or other features, and point features may be central points or end points, etc.

Single features may be combined to form higher level features such as slots, pockets, steps and notches (sec figure 1). These combinations are commonly seen in practice and which may have tolerances of position or symmetry defined with respect to their median planes, axes or central lines.

\section{Datums and datum features}

Dimensions and tolerances are normally applied with respect to feature datums (except for tolerances of form). A datum is a theoretically exact geometric reference such as a centre point, an axis, a centre line, a plane face or a median plane. Since theoretically perfect surfaces cannot be produced, surface plates, machine tables, axes or median planes of gauges and other equipment used in manufacturing and inspection are usually sufficiently accurate that they may be considered theoretical planes and axes and thus used as datums. A feature of a component in contact with a datum is called the datum feature. Datum features should be chosen by considering the function of the part and with high accuracy. Therefore, form tolerances such as straightness, flatness, cylindricity and roundness are normally required to ensure the accuracy or datum features. 
Datum systems

For manufacturing and inspection purposes, a three-plane datum system is required for most non-rotational parts, so that the orientation may be defined and the parts can be located with respect to the datum systems of machining or measuring machines. The three datum planes are mutually perpendicular and are known as the primary, secondary and tertiary datum surface respectively. The primary datum plane is defined by three points contacting the face or the part, and these are known as datum targets. The secondary datum plane is defined by two contacting points and the tertiary datum plane is defined by one point.

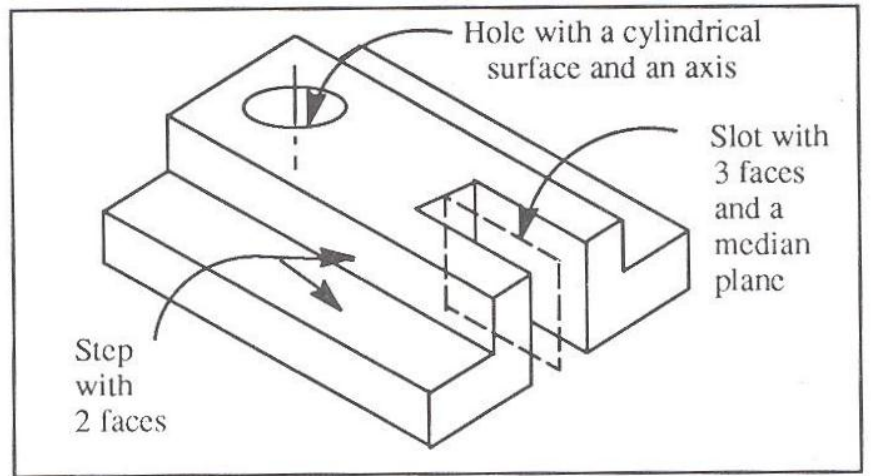

Figure 1. Features used for Tolerancing

\section{Current Geometric Modellers and Tolerancing}

As discussed above, the specification or dimensions and tolerances requires a featurebased component representation. However, current geometric modellers are not featurebased. Although Brep models contain detailed information about edges, faces and vertices of components which are essential for tolerance definition, such elements are not distinctively represented as features. For example, during interactive design and editing, the names of the geometric elements of a component will change arbitrarily, especially when Boolean operations are performed.

The latest parametric systems such as Pro-engineer (James, 1991) and CADDS (Computervision, 1991) are able to represent a number of commonly used features such as slots, pockets, holes, steps, etc. Dimensions are represented and manipulated as parameters. However, lower-level features such as points, lines and surfaces are still not represented as distinctive features. In fact, the parametric feature data is stored separate from a Brep model of the same component (although the two data models are associated with each other to certain extent). The former doesn't contain sufficiently detailed information for tolerancing, whilst, the later suffers from the same problem found with all Brep models.

\section{Feature-based Component Representation}

Since the current geometric modellers (even the latest parametric systems) are unable to support feature-based product data models for dimension and tolerance definition, an alternative representation has been proposed and implemented in a prototype feature-based design system. Three levels of features are defined, which are:

ATOMIC FEATURES, which are the lowest level features, i.e. points, lines and 
surfaces. Point features may be central points or vertices of lines or other features. Line features may be centre lines, axes or edges. Surface features may be real faces, median planes, etc. Atomic features are essential for all types of tolerances. Atomic features are usually treated as the basic constituent elements of higher level features.

PRIMITIVE FEATURES, which are at the second level, are groups of atomic features which form recognisable geometric entities such as holes, pockets, slots, notches and step. These are commonly described as functional or manufacturing features. A primitive feature can be toleranced by positional and symmetrical tolerances with respect to its axis or median plane(s). Its constituent atomic features can be toleranced or used as datums to tolerance other features.

COMPOUND FEATURES are the top level of features, e.g. patterns of holes, counter-bores, multi-steps, crossed slots. A compound feature is a combination of primitive features and/or atomic features which together perform a single function or may be manufactured by a set of operations. Usually the constituent features, rather than the compound feature, are used for tolerancing.

Using the above feature definition, a component, and hence a product can be modelled and functional dimensions and tolerances can be specified with respect to atomic features and primitive features. The primary advantage of this representation is that the three levels of features are inter-related in a hierarchical fashion, whilst the distinctiveness and consistency of individual features are maintained.

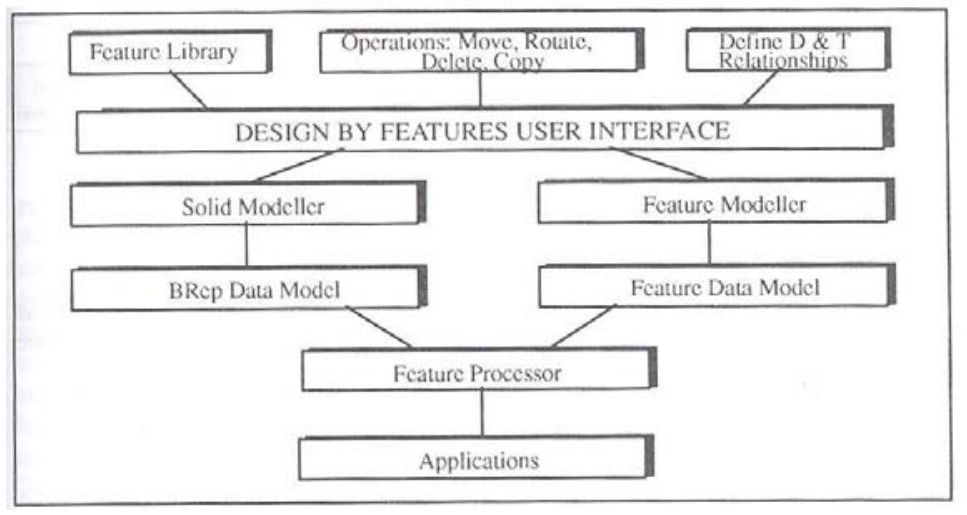

Figure 2. The Feature-based Design System

\section{Implementation}

The feature-based component representation has been implemented in LUT-FBDS (Loughborough University of Technology Feature-Based Design System) (Case et al, 1994). The system consists of a user interface, a Brep solid modeller, a feature modeler and a feature processor (figure 2). The primitive features available in the feature library are boss, pocket, hole, through slot, non-through slot, notch, step and surface. Atomic features are not pre-defined in the feature library. However, they are represented as constituent entities of primitive features and their consistency and independence are maintained in the feature data model. For example, a through hole (which is an instance of the hole primitive) is composed of two atomic features, a cylindrical face and an axis. The axis is normally used for tolerancing. Note that for process planning we also define two imaginary faces at the two ends of the hole, but they are not treated as atomic features. 
A number of compound features are also pre-defined in the feature library, including pattern holes, cross-slot, counterbore and multi-step. More compound features can be defined by combining primitive features through the user interface. In the database, compound features are treated as relationships between primitive features. Dimensions and tolerances can be defined through the menu-based user interface, which comply with the British Standard BS 308 (BSI, 1988) as shown in figure 3. As the consistency of lower level features are maintained in the database, the validity of the specification of dimensions and tolerances are assured for the whole life cycle of the product.

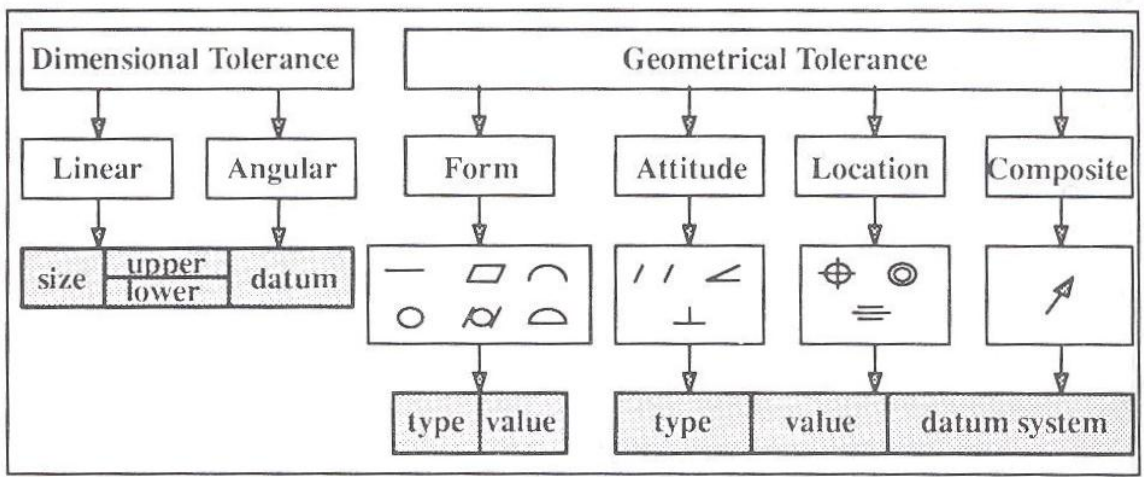

Figure 3. Tolerances that can be defined through the user interface

\section{Conclusions}

The geometric elements required for dimension and tolerance definition are features, including primitive features, and more importantly, lower level features such vertices, axes, edges, lines, surfaces and median planes. Current geometric modellers are not feature-based or are unable to represent lower level features. Therefore, tolerance information can only be either represented as text symbols without implication in the database, or if stored in the database, the consistency cannot be maintained as the identifiers of the lower level geometric elements (features) of components cannot be maintained. The implemented feature-based representation overcomes this problem to a significant extent.

\section{References}

BSI, 1988, Manual or British Standards in Engineering Drawing and Design, 2nd edn (British Standards Institution, Maurice Parker ed).

Case, K., Gao, J. X. and Gindy, N. N. Z. 1994, The implementation of a feature-based component representation for CAD/CAM integration, the Proceedings of IMechE, Part 2, Journal of Engineering Manufacture, 208, 71-80.

Computervision, 1991, Introduction to CADDS 5 Parametric Design (Tutorial ), ( Prime Computervision, DOC38800-1 LA).

James, M. July 1991, Prototyping Cad, cover story of CADCAM.

Juster, N. P. 1992, Modelling and representation of dimensions and tolerances: a survey, Computer-Aided Design, 24 (1), 3-17.

Mantyla, M. 1988, An introduction to solid modelling, (Computer Science Press, Maryland, USA). 